\title{
Bluetooth-enabled Activity Monitor
}

National Cancer Institute

\section{Source}

National Cancer Institute. Bluetooth-enabled Activity Monitor. NCI Thesaurus. Code C150419.

A consumer, wearable device that is designed to collect and display information about an individual's activities and is able to transfer that information to another device or system over a Bluetooth connection. Information collected may include steps, stairs climbed, distance traveled, calories used, heart rate, sleep quality, or other metrics. 